\title{
ARE BINARY SEPARATIONS RELATED TO THEIR SYSTEM MASS?
}

\author{
Michael F. Sterzik ${ }^{1}$ and Richard H. Durisen ${ }^{2}$ \\ RESUMEN
}

Listamos los datos más recientes sobre la fracción de multiplicidad y la distribución de separaciones de binarias para distintas masas de la primaria, incluyendo primarias de muy baja masa y enanas marrones, y las comparamos con modelos dinámicos de disolución de cúmulos con $N$ pequeño. Las predicciones de los modelos están basadas en cálculos numéricos detallados de la dinámica interna de los cúmulos así como en métodos tipo Monte Carlo. Tanto las observaciones como los modelos muestran las mismas tendencias: (1) La fracción de multiplicidad aumenta con la masa de la primaria. (2) La separación media de las binarias aumenta con la masa del sistema, de tal forma que las binarias de muy baja masa tienen separaciones medias del orden de $4 \mathrm{AU}$, mientras que la separación de las binarias de una masa solar tiene un máximo alrededor de $40 \mathrm{AU}$. Las binarias de tipo M pueblan preferentemente las separaciones intermedias. Esta tendencia puede quizás explicarse si la energía específica al momento de formarse el cúmulo es similar para cúmulos de distintas masas.

\begin{abstract}
We compile most recent multiplicity fractions and binary separation distributions for different primary masses, including very low-mass and brown dwarf primaries, and compare them with dynamical decay models of small$N$ clusters. The model predictions are based on detailed numerical calculations of the internal cluster dynamics, as well as on Monte-Carlo methods. Both observations and models reflect the same trends: (1) The multiplicity fraction is an increasing function of the primary mass. (2) The mean binary separations are increasing with the system mass in the sense that very low-mass binaries have average separations around $\approx 4 A U$, while the binary separation distribution for solar-type primaries peaks at $\approx 40 A U$. M-type binary systems apparently preferentially populate intermediate separations. Similar specific energy at the time of cluster formation for all cluster masses can possibly explain this trend.
\end{abstract}

\section{Key Words: BINARIES: GENERAL - STARS: FORMATION - STARS: PRE-MAIN SEQUENCE}

\section{INTRODUCTION}

Many, if not most, stars are observed as members of binary and multiple systems. Their properties give critical constraints on their formation and evolution history. However, the detailed mechanisms that define the binary fraction, period and mass ratio distributions remain to be understood, and modeled.

Nearby solar-type dwarfs serve as a well-studied sample for which both the multiplicity fractions as well the separation distributions are measured over a large, continuous period range (Duquennoy \& Mayor, 1991). The broad, log-normal shape of the period distribution is frequently used as a reference distribution for comparisons. To what extent does this characterize different populations? Differences in primary mass, age, evolutionary phase, or formation environment are likely to imprint different binary or multiple star properties.

Recent observational advances include the determination of the multiplicity fractions along a broad

\footnotetext{
${ }^{1}$ European Southern Observatory, Chile.

${ }^{2}$ Department of Astronomy, Indiana University, USA.
}

range of primary masses. The application of adaptive optics techniques on faint reference stars allows us to search for close companions among very-low mass (VLM) and therefore faint primary stars, even across the sub-stellar limit. Interestingly, these observations indicate that (1) the binary (or multiplicity) fraction among the low-mass stars appears to be lower and (2) the separation distribution appears to be narrower and peaked towards smaller values than for solar-type stars. Therefore we concentrate in this contribution on the influence of the system mass on the properties of binary or multiple stars. We will discuss the relevant observations especially towards the lower end of the stellar mass function, and will sketch how these observations can be interpreted in the framework of dynamical decay models. For a thorough description of the approach and the models we refer to Sterzik \& Durisen (2003), and references therein, where we give a detailed analysis of the imprints of dynamical interactions on pairing statistics and kinematics, with special emphasis on brown dwarfs. 


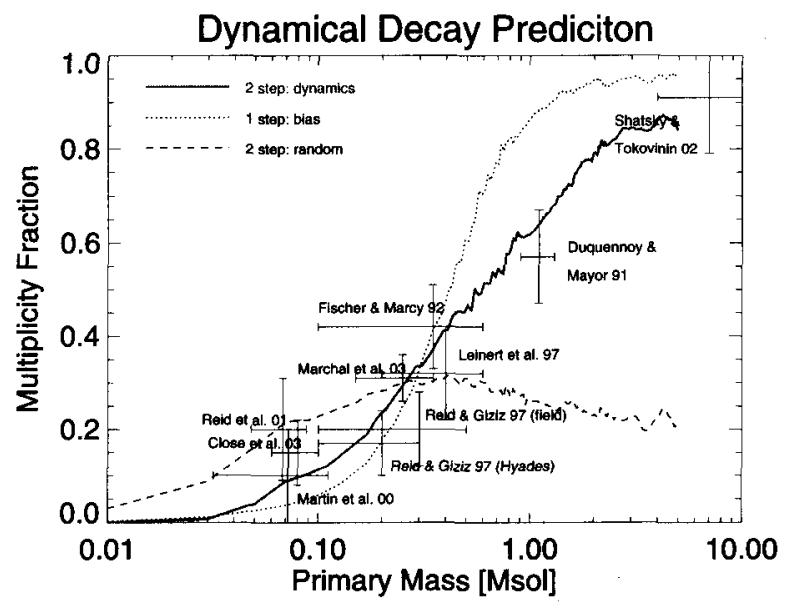

Fig. 1. Multiplicity fraction dependence on primary mass. We compare observations and three models prescriptions.

\section{MULTIPLICITY FRACTIONS}

Let us first consider multiplicity fractions $(\mathrm{MuF})$. We define the MuF in the usual way as the sum of binary and all higher order multiple systems divided by the sum of all systems, i.e. single stars, binaries and higher order systems. In Fig. 1 we display MuF versus the primary mass obtained from different authors. The data points represent, to our knowledge, those observations of $\mathrm{MuF}$ for different primary mass ranges that represent statistically meaningful samples. We try to estimate fair primary mass ranges and $\mathrm{MuF}$ errors from the original references, if they are not directly given. In particular we refer to the following studies, in ascending order of primary mass: Reid et al. (2001), HST search for L-dwarf binary systems; Martin et al. (2000), Multiplicity of VLM stars in the Pleiades; Close et al. (2003), Adaptive Optics search for VLM binaries; Reid \& Gizis (1997a), Low-mass binaries in the field; Reid \& Gizis (1997b), Low-mass binaries in the Hyades; Marchal et al. (2003), Multiplicity among late M-dwarfs; Leinert et al. (1997), Near-IR speckle interferometry of nearby southern M-dwarfs; Fischer \& Marcy (1997), Multiplicity among M-dwarfs; Duquennoy \& Mayor (1991), Multiplicity among solar-type stars; Shatsky \& Tokovinin (2002), B-type binaries in Sco OB2.

We note a steady and continuous increase of the observed MuF with primary mass. The MuF in the VLM regime is significant lower than for solar-type masses. At the brown-dwarf mass boundary, the fraction ranges from 10 to $20 \%$, but the measurements are affected by relatively large statistical error bars. The most recent determination of Close et al. (2003) has the highest statistical significance. Their value of $15 \pm 7 \%$ refers to a mass range approximately between 0.06 and $0.1 M_{\odot}$. On the highmass end, we refer to the value reported in Shatsky \& Tokovinin (2002), and extrapolate their value to the entire separation regime. Other studies for $F$, $\mathrm{A}$, and $\mathrm{B}$ type stars appear to corroborate the high fraction of binaries around these spectral types (see, e.g., Kouwenhoven et al., 2004).

The different curves in Fig. 1 refer to different classes of dynamical decay models, which are explained in detail in Sterzik \& Durisen (2003). Here we will briefly summarize the basic assumptions and methodology. One basic assumption for all model classes is the underlying initial mass function (IMF), and how the stars obtain their masses within their initial clusters. We assume an IMF that is as closely as possible constrained by observational data. For stellar masses, the IMF is very similar to the one derived by Kroupa (2001) for the galactic field. The sub-stellar regime is not as well known, but an index of $0.5<\alpha<1$ for the mass function $d N / d M \propto M^{-\alpha}$ appears to hold for the field as well as for young open clusters (see, e.g., Chabrier 2002). We choose $\alpha=0.5$, and set the lower and higher mass limit to $0.01 M_{\odot}$ and $10 M_{\odot}$, resp.

Next, we compare three distinct model classes for how individual stars form in clusters and later decay. These models span a range of plausible alternatives. For each case, we apply a Monte-Carlo method (explained in Durisen, Sterzik \& Pickett 2001) to generate a large number of ensembles and to draw statistically significant conclusions. The most simple model assumes "complete dynamical biasing". This means that each cluster will eventually decay into one stable binary, consisting of the two most massive members. This assumption is reasonable, provided stellar dynamics alone governs the dynamical evolution, and no dissipation and/or hierarchical fragmentation constraints are taken into account. If the stellar masses are then drawn randomly from an IMF without other constraint, we obtain a final pairing statistics (or MuF) that is indicated with the dotted curve. Except for an intermediate mass range, the curve appears to be too steep, and incompatible with observations. This corroborates the analysis of McDonald \& Clarke (1993), who studied this scenario analytically, with a similar conclusion.

The dashed curve refers to an alternative, extreme scenario, where stars are paired at random among cluster members. The physical interpretation of this scenario is that strong dissipation dominates the dynamical evolution. In this case, the MuF curve 
is relatively smooth, reflecting the fact that the likelihood of obtaining low mass pairs is similar to obtaining pairs with high mass. Although the low mass $\mathrm{MuF}$ is boosted, in accord with observations, the deviations for higher mass primaries are unacceptable.

The full line refers to a third scenario. In Sterzik $\&$ Durisen (1998) we introduced a novel concept concerning how stellar clusters are formed within fragmenting clouds. If we assume that cloud cores are the precursors of the stellar clusters, and that the cloud core mass spectrum itself resembles a stellar mass spectrum, then we can assume that the sum of the stellar masses in each cluster is constrained by its precursor core mass. We have implemented this notion in our models, and refer to it as the "two-step IMF" approach, as it involves independent stellar and core mass functions (Durisen, Sterzik \& Pickett 2001). The additional constraint on the cluster masses results in a more uniform distribution of stellar masses within each cluster, while still matching the overall stellar IMF. We also drop the condition of "complete dynamical biasing", because there are significant deviations from the simple prescription that only the two most massive stars form a stable pair within a given cluster. Instead we assume pairing weights that have been determined from detailed numerical integrations of the dynamical decay.

We conclude that the "two-step" scenario gives a very good match to the observed MuF, especially for the stellar mass range. It is inconclusive from current data whether the "two-step" scenario describes the $\mathrm{MuF}$ for brown dwarf masses correctly. A high brown-dwarf binary frequency will require the action of dissipation during formation and/or a higher initial fraction of brown dwarfs (i.e. a higher value of $\alpha)$.

\section{SEPARATION DISTRIBUTIONS}

We will now discuss recent observations of binary separations having different system (or primary) mass. Therefore, we plot in Fig. 2 the cumulative distribution function of separations compiled for different system masses. For comparison we refer to the separation distribution for solar-type dwarfs, approximated as a log-normal distribution using a mean separation and width of $\log \Delta=\log \sigma=1.5$ (Duquennoy \& Mayor 1991, thin full line). Much narrower, and at lower mean separations, the cumulative separation distribution of 34 VLM and browndwarf binaries stems from Close et al. (2003) and is drawn as a dashed line. It can be well approximated by a $\log$-normal distribution, with $\log \Delta=\log \sigma=$ 0.5. (also shown as a thin dashed line). The dashed-

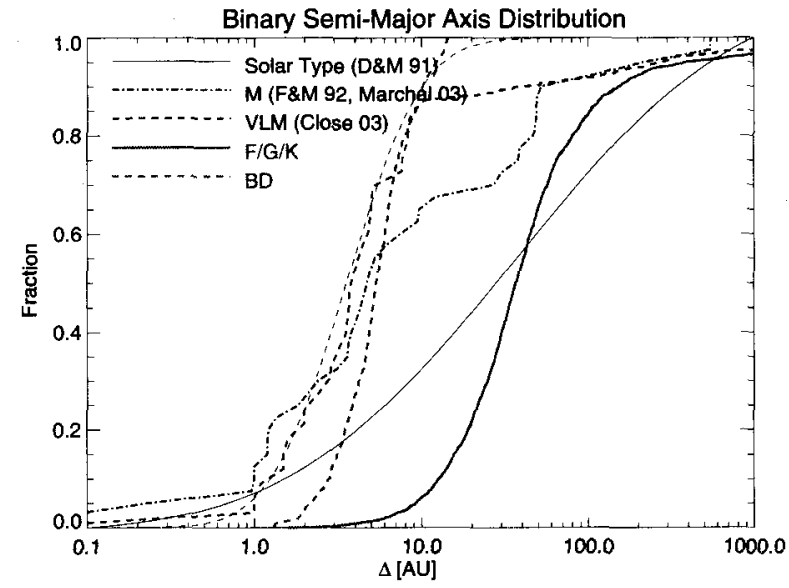

Fig. 2. Cumulative separation distributions for samples of different system mass.

dotted line consists of 56 late M-dwarfs in the spectral type range approximately ranging from M2 to M6. It is constructed from the lists published by Fischer \& Marcy (1997) and Marchal et al. (2003), and is located intermediate between the VLM objects, and the solar-type binaries. We conclude that these data suggest a significant dependence of the binary separation distribution with mass, in the sense that lighter systems tend to have smaller mean separations and narrower distributions.

We will now try to explain these trends in the framework of the dynamical decay modelling. Motivated by the fact that the "two-step" IMF approach reproduces the observed trends in the MuF (and the mass ratio distributions, too) we elaborate an argument on how the system scale of the stellar cluster relates to the physical scale of the cloud-core precursor. Let us assume that these precursor clumps of the few-body stellar systems resemble radial density profiles of the Bonnor-Ebert type, as e.g. observed for certain types of Bok globules (Alves et al. 2001). In these clumps the mass $M_{c}$ versus size $R_{c}$ relation is of linear form, in agreement with observations from Motte et al. (2001). This relation also implies that the specific energy in star forming clumps is constant over the observed mass and size ranges. Hydrodynamical simulations of the protostellar collapse have shown that these flattish radial density profiles can fragment into several protostars, in contrast with the density profiles known from isothermal, spherical collapse. We can therefore assume that a certain number of precursor clumps actually produce few-body stellar systems. Unfortunately, the system scale of the initial few-body stellar cluster is much more difficult to predict. The detailed hydro- 
and thermodynamics involved in the physics of stellar collapse will determine the stellar system scale. To advance the argument, it appears reasonable to assume that a certain fraction of the initial clump mass is actually converted in stellar mass by a (constant) star formation efficiency. Moreover, we might speculate that the clump scale $R_{c}$ and the stellar cluster size are also linearly related, e.g. when several Jeans masses (say, $N$ ) of clump gas convert into stars. With these assumptions, the specific energy of stellar systems stays constant for all scales. This has the important consequence that the virial speed $v_{v i r}$ for all few-body clusters is constant. In Sterzik \& Durisen (1998) we have then explored the relation of the expected dispersion velocities and binary separations on this scaling parameter, and determined its approximate value from plausible star formation arguments to be $v_{v i r}=3.3 \mathrm{~km} \mathrm{~s}^{-1}$.

In Fig. 2 we overplot the separation distribution for binaries obtained from direct, numerical, dynamical decay simulations applying the physical scaling as described above. The thick, full line corresponds to a sample of solar-type binaries, whereas the thick, dotted line corresponds to the sub-stellar mass bin. Strikingly, we recover not only that the mean separations scale like the observations, but also that the mean values are actually very close to the observed values. On the other hand, the shape of the distribution function is different from the observed one, especially for the solar-type binaries. In other words, the pure dynamical cluster simulations cannot account for the broad separation distribution of solar-type binaries. Other evolutionary process must play a role in broadening this distribution (see, e.g. Valtonen, 2004). On the other hand, the observed width of the VLM binary separation distribution is similar to the simulated one.

\section{CONCLUSIONS}

We have compiled recent observations of multiplicity fractions and binary separations for different spectral types (i.e. masses). We recover two firm trends: (1) The multiplicity fraction is an increasing function of the primary mass. (2) The mean binary separations (and distribution widths) increase with system mass. Observation (1) can be best explained by dynamical decay of few-body clusters, assuming a "two-step" process where few-body clusters form as products of hierarchical fragmentation in cloud cores. Observation (2) is also consistent with the formation of binaries as end-products of dynamically decaying stellar systems, provided the initial stellar clusters have constant specific energy.

M.F.S. acknowledges the use of separations for late M-dwarfs from Lydie Marchal before publication, as well as the inspiring atmosphere at the meeting.

\section{REFERENCES}

Alves, J. F., Lada, C. J., \& Lada, E. A. 2001, Nature, 409, 159

Chabrier, G. 2002, ApJ, 567, 304

Close, L. M., Siegler, N., Freed, M., \& Biller, B. 2003, ApJ, 587, 407

Durisen, R. H., Sterzik, M. F., \& Pickett, B. K. 2001, A\&A, 371, 952

Duquennoy, A., \& Mayor, M. 1991, A\&A, 248, 485

Fischer, D. A., \& Marcy, G. W. 1992, ApJ, 396, 178

Kouwenhoven, M. B. N., Brown, A. G. A., Gualandris, A., Kaper. L., Portegies Zwart, S. F., \& Zinnecker, H. 2004, this volume

Kroupa, P. 2001, MNRAS, 322, 231

Leinert, C., Henry, T., Glindemann, A., \& McCarthy, D. W., Jr. 1997, A\&A, 325, 159

Marchal, L., Delfosse, X., Forveille, T., Ségransan, D., Beuzit, J. L., Udry, S., Perrier, C., Mayor, M., \& Halbwachs, J.-L. 2003, in IAU Symp. 211, Brown Dwarfs, ed. E. Martin (San Francisco: ASP), 311

Martin, E. L., Brandner, W., Bouvier, J., Luhman, K. L., Stauffer, J., Basri, G., Zapatero Osorio, M. R., \& Barrado y Navascués, D. 2000, ApJ, 543, 299

McDonald, J. M., \& Clarke, C. J. 1993, MNRAS 262, 800

Motte, F., André, P., Ward-Thompson, D., \& Bontemps, S. 2001, A\&A, 372, 41

Reid, I. N., \& Gizis, J. E. 1997a, AJ, 113, 2246

Reid, I. N., \& Gizis, J. E. 1997b, AJ, 114, 1992

Reid, I. N., Gizis, J. E., Kirkpatrick, J. D., \& Koerner, D. W. 2001, AJ, 121, 489

Shatsky, N., \& Tokovinin, A. 2002, A\&A, 382, 92

Sterzik, M. F., \& Durisen, R. H. 1998, A\&A, 339, 95

Sterzik, M. F., \& Durisen, R. H. 2003, A\&A, 400, 1031

Valtonen, M. 2004, this volume

Richard H. Durisen: Department of Astronomy, SW319, Indiana University, Bloomington, Indiana 47405,

U.S.A. (durisen@astro.indiana.edu).

Michael F. Sterzik: European Southern Observatory, Casilla 19001, Santiago 19, Chile (msterzik@eso.org). 


\section{DISCUSSION}

Tokovinin - If the separation distribution of G-dwarfs is broadened by wide variation of specific energy of clouds, why is the distribution of VLM stars so narrow?

Sterzik - For the simulations shown we used strictly constant specific energy for the initial stellar clusters. Naturally, relaxing this condition broadens the resulting separation distributions. Observationally the VLM stars appear to originate from a much narrower initial specific energy distribution than do higher mass clusters.

Tokovinin - There seems to be an apparent discrepancy between the narrow separation distributions that you obtain from your simulations and the wider distribution that was obtained by C. Allen and A. Poveda. Please comment.

Sterzik - Allen and Poveda used much broader initial configuration distributions.

Clarke - You get the mass versus separation correlations purely from assuming a constant Jeans number in the progenitor clumps. This would work fine if each clump fragmented into $\mathrm{N}=2$ stars, i.e., it does not rely on dynamical interactions at all. How important are these interactions in the production of VLM binaries in your models?

Sterzik - In our model, about $1 / 2$ of all BD (VLM) binaries actually stem from N = 2 progenitors; in these cases dynamical interactions play no role. The other half is affected; we find a typical shrinkage of the system scale by a factor of 5 from the initial separations towards the final (binary) separations due to dynamical encounters.

Mathieu - While the small-N dynamical mechanism produces the long-period frequency distribution, the frequency distribution found by Duquennoy \& Mayor is unimodal and does not suggest distinct period regimes. Can your modeling naturally produce the entire period distribution?

Sterzik - Whereas we can reproduce the mean of the separation (or period) distributions for different primary masses, we cannot account for the broad width of the Duquennoy-Mayor distribution by pure N-body dynamics processes.

Bate - In relation to forming spectroscopic binaries, arbitrary amounts of orbital angular momentum can be removed from a binary by a circumbinary disc, so I don't think forming spectroscopic binaries is necessarily a problem.

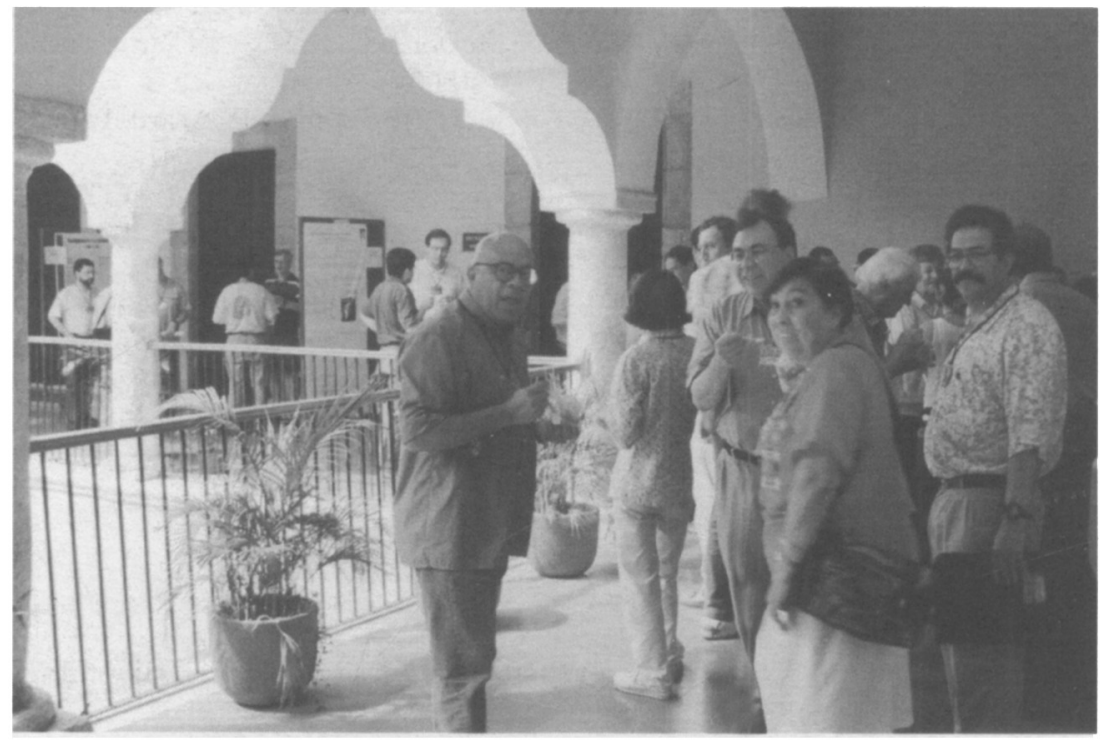

José Peña, Jesús Galindo, M. G. Saucedo and Salvador Cuevas. 